\title{
Downward trend in pediatric resident laryngoscopy participation in pediatric ICUs
}

\author{
Aayush Gabrani, MD¹, Taiki Kojima, MD¹, Ronald C. Sanders Jr., MD, MS², Asha Shenoi, \\ MD, FAAP ${ }^{3}$, Vicki Montgomery, MD, FCCM ${ }^{4}$, Simon J Parsons, MD $^{5}$, Sandeep Gangadharan, \\ MD $^{6}$, Sholeen Nett, MD, PhD ${ }^{7}$, Natalie Napolitano, MPH, RRT-NPS, FAARC ${ }^{8}$, Keiko \\ Tarquinio, $\mathbf{M D}^{9}$, Dennis W. Simon, MD ${ }^{10}$, Anthony Lee, MD ${ }^{11}$, Guillaume Emeriaud, MD ${ }^{12}$, \\ Michelle Adu-Darko, MD, FAAP ${ }^{13}$, John S. Giuliano Jr., MD ${ }^{14}$, Keith Meyer, MD ${ }^{15}$, Ana Lia \\ Graciano, MD, FAAP, FCCM ${ }^{16}$, David A. Turner, MD ${ }^{17}$, Conrad Krawiec, MD ${ }^{18}$, Adnan M \\ Bakar, MD ${ }^{6}$, Lee A Polikoff, MD, FAAP ${ }^{9}$, Margaret Parker, MD ${ }^{19}$, llana Harwayne-Gidansky, \\ MD $^{19}$, Benjamin Crulli, MD ${ }^{12}$, Paula Vanderford, MD, FAAP ${ }^{20}$, Ryan K. Breuer, MD ${ }^{21}$, Eleanor \\ Gradidge, MD 22 , Aline Branca, MD22, Lily B. Grater-Welt, MD ${ }^{6}$, David Tellez, MD ${ }^{22}$, Lisa V. \\ Wright, MHA, RRT-NPS, AE-C ${ }^{23}$, Matthew Pinto, MD ${ }^{24}$, Vinay Nadkarni, MD ${ }^{25}$, and Akira \\ Nishisaki, MD, MSCE ${ }^{25}$ for the National Emergency Airway Registry for Children \\ (NEAR4KIDS) collaborators and Pediatric Acute Lung Injury and Sepsis Investigators \\ (PALISI) \\ ${ }^{1}$ Center for Simulation, Advanced Education and Innovation, Children's Hospital of Philadelphia, \\ Philadelphia, PA, USA \\ ${ }^{2}$ Department of Pediatrics, Section of Pediatric Critical Care, UAMS/Arkansas Children's Hospital, \\ Little Rock, AR, USA \\ ${ }^{3}$ Department of Pediatrics, Section of Pediatric Critical Care, Kentucky Children's Hospital, \\ University of Kentucky, Lexington, KY, USA \\ ${ }^{4}$ Pediatric Critical Care, Norton Children's Hospital, University of Louisville, Louisville, KY, USA \\ ${ }^{5}$ Department of Pediatrics, Section of Critical Care Medicine, Alberta Children's Hospital, Calgary, \\ $\mathrm{AB}$, Canada
}

Corresponding Author: Akira Nishisaki, MD, MSCE, Department of Anesthesiology and Critical Care Medicine, Children's Hospital of Philadelphia, 3401 Civic Center Blvd. CHOP Main 8566, Philadelphia, PA 19106, USA, Nishisaki@email.chop.edu.

Disclosure of prior publications:

This study was designed to follow up our previous study published in year 2013: Sanders RC, Giuliano JS, Sullivan JE, Brown CA, Walls RM, Nadkarni V, et al. Level of trainee and tracheal intubation outcomes. Pediatrics [Internet]. 2013;131(3):e821-8. The study objectives, design, and results are substantially different.

The rest of authors have no conflicts of interest relevant to this article to disclose.

Clinical Trial Registration: NCT02493478

Copyright form disclosure: Dr. Napolitano's institution received funding from research Relationships with Draeger, Aerogen, Smiths Medical, GeNO, Philips Respironics, and actuated Medical and from grant support R21 and R18 from NICHND and Agency for Healthcare Research and Quality (AHRQ). She received funding from reimbursement for travel only for board positions for American Association for Respiratory Care and Allergy and Asthma Network. Dr. Emeriaud's institution received funding from a young investigator grant of the Respiratory Health Network of the Fonds de Recherche du Quebec-Santé and from a Clinical Research Scholarship of the Fonds de Recherche du Quebec - Sante. Dr. Polikoff received funding from Roche Pharmaceuticals. Dr. Parker received funding from Raynes McCarty. Dr. Glater-Welt received funding from the National Institutes of Health. Dr. Nishisaki institution received funding from AHRQ R03HS021583, R18HS022464, and R18HS024511, and he received support for article research from the AHRQ. The remaining authors have disclosed that they do not have any potential conflicts of interest. 
${ }^{6}$ Department of Pediatrics, Division of Critical Care Med, Cohen's Children's Medical Center, New Hyde Park, NY, USA

${ }^{7}$ Pediatric Critical Care, Dartmouth-Hitchcock Medical Center, Lebanon, NH, USA

${ }^{8}$ Respiratory Care Department, Children's Hospital of Philadelphia, Philadelphia, PA, USA

${ }^{9}$ Pediatric Critical Care Medicine, Department of Pediatrics, Brown University Hasbro Children's Hospital

${ }^{10}$ Department of Critical Care Medicine, Children's Hospital of Pittsburgh of UPMC

${ }^{11}$ Critical Care Medicine, Nationwide Children's Hospital

${ }^{12}$ Department of Pediatrics, CHU Sainte Justine, Université de Montréal

${ }^{13}$ Pediatric Critical Care Department, University of Virginia Hospital

${ }^{14}$ Pediatric Intensive Care Unit, Section of Pediatric Critical Care, Yale University School of Medicine

${ }^{15}$ Division of Critical Care, Miami Children's Hospital

${ }^{16}$ Pediatric Critical Care Medicine, University of Maryland School of Medicine

${ }^{17}$ Division of Pediatric Critical Care, Department of Pediatrics, Duke Children's Hospital

${ }^{18}$ Pediatric Critical Care, Department of Pediatrics, Penn State Children's Hospital

${ }^{19}$ Pediatric Critical Care Medicine, Stony Brook Children's Hospital

${ }^{20}$ Pediatric Critical Care, Oregon Health and Science University

${ }^{21}$ Division of Critical Care Medicine, Department of Pediatrics, Women's and Children's Hospital of Buffalo

${ }^{22}$ Division of Pediatric Critical Care, Phoenix Children's Hospital

${ }^{23}$ Respiratory Therapy, Kentucky Children's Hospital, UK HealthCare

${ }^{24}$ Department of Pediatrics, Division of Pediatric Critical Care Medicine, Maria Fareri Children's Hospital, NY, USA

${ }^{25}$ Department of Anesthesiology and Critical Care Medicine, Children's Hospital of Philadelphia, Philadelphia, PA, USA

\section{Abstract}

Objective-As of July 2013, pediatric resident trainee guidelines in the United States (US) no longer require proficiency in non-neonatal tracheal intubation (TI). We hypothesized that laryngoscopy by pediatric residents has decreased over time, with a more pronounced decrease after this guideline change.

Design-Prospective cohort study

Setting -25 pediatric ICUs at various children's hospitals across United States of America 
Patients-Tracheal intubations performed in PICUs from July 2010 to June 2016 in the multicenter TI database (National Emergency Airway Registry: NEAR4KIDS)

Intervention-None

Measurements and Main results-Prospective cohort study in which all primary TIs occurring in the US from July 2010 to June 2016 in the multicenter TI database (National Emergency Airway Registry: NEAR4KIDS) were analyzed. Participating pediatric ICU leaders were also asked to describe their local airway management training for residents. Resident participation trends over time, stratified by presence of a pediatric critical care medicine (PCCM) fellowship and airway training curriculum for residents, were described. 9,203 TIs from 25 PICUs were reported. Pediatric residents participated in 16\% of TIs as first laryngoscopists: $14 \%$ in pediatric ICUs with a PCCM fellowship and $34 \%$ in pediatric ICUs without one $(\mathrm{p}<0.001)$. Resident participation decreased significantly over time (3.4\% per year, $\mathrm{p}<0.001)$. The decrease was significant in ICUs with a PCCM fellowship $(\mathrm{p}<0.001)$ but not in ICUs without one $(\mathrm{p}=0.73)$. After adjusting for site-level clustering, patient characteristics and PCCM fellowship presence, the ACGME guideline change was not associated with lower participation by residents (Odds Ratio: $0.86,95 \%$ CI $0.59-1.24, \mathrm{p}=0.43$ ). The downward trend of resident participation was similar regardless of the presence of an airway curriculum for residents.

Conclusion-Laryngoscopy by pediatric residents has substantially decreased over time. This downward trend was not associated with the 2013 ACGME change in residency requirements.

\section{Keywords}

tracheal intubation; ACGME; resident; pediatric ICU

\section{INTRODUCTION}

Tracheal intubation (TI) is a life-saving procedure in critically ill infants and children (1). Critically ill children often have challenging airway anatomy, low oxygen reserve, or lifethreatening hemodynamic instability that places them at high risk for TI-associated adverse events. These challenges, coupled with the low frequency of pediatric trainee exposure to airway management, make TI procedural competency difficult to achieve.

Published TI participation and success rates of pediatric residents have been suboptimal: approximately $20 \%$ to $30 \%$ for participation and $30 \%$ to $50 \%$ for success, respectively (13 ). Our previous study across 15 pediatric intensive care units (PICUs) showed that residentlevel provider TI success is lower and that adverse TI associated events are significantly higher, when compared with fellow-level providers (2). These findings were thought to be due to limited exposure and lack of structured airway management training for pediatric resident trainees.

Pediatric residents are expected to achieve competence in specific procedures by the end of residency training in the United States (US) (4). A recent update in the Accreditation Council for Graduate Medical Education (ACGME) guidelines (effective from July 2013) eliminated the requirement for non-neonatal TI, while retaining the requirement of neonatal TI proficiency (5). 
With the recent change in ACGME guidelines eliminating non-neonatal TI requirement, both resident participation and success rates in PICU TIs may have substantially declined. However, since the 2013 ACGME guideline change, there has been limited information describing the trend of pediatric resident TI participation and first attempt success rates in a wide range of PICUs across the US. Given that the local needs for pediatricians' technical skills are variable across the US, it is possible that some pediatric residency programs continue training for non-neonatal TIs.

The study's primary aim is to describe the trend of pediatric resident TI participation over time and first attempt success rates in a wide range of PICUs across the US using an existing large quality improvement TI safety database: National Emergency Airway Registry for Children (NEAR4KIDS). This study also aims to explore the association between resident participation and training structure, including the presence of a PCCM fellowship and the existence of an airway management training curriculum for residents.

Our specific hypothesis was that the participation of pediatric residents in TIs as first laryngoscopists has declined over time, and that the 2013 ACGME residency requirement change significantly impacted the participation rate. Secondary hypotheses were that the decline in pediatric resident participation would be more pronounced in PICUs with PCCM fellowship, and would be less prominent in PICUs with an existing airway management training curriculum for residents.

\section{MATERIALS AND METHODS}

\section{Study Design}

This is a retrospective multicenter cohort study in 25 PICUs with pediatric residency training programs across the US from July 2010 to June 2016 (6 Fiscal Years [FY]). Institutional Review Board (IRB) approval or exempt from review status was obtained at each participating site. For the additional questions regarding resident airway management training curriculum and expectations for TI procedure, IRB exemption was granted at the data coordinating center for this study.

\section{Data collection}

Multicenter TI data-A de-identified dataset was obtained from the prospective multicenter observational cohort of TIs that were reported to the NEAR4KIDS quality improvement project. The NEAR4KIDS database was created by members of the Pediatric Acute Lung Injury and Sepsis Investigators (PALISI) Network (1, 6-14). Collected TI variables included patient characteristics (such as demographics and TI indications), provider characteristics, TI method details, process of care variables (i.e., number of attempts), and TI outcomes (i.e., first attempt success). Data collection was standardized with operational definitions for each data point. Each site developed a compliance plan to ensure accuracy, complete data capture, and timely data entry $(1,6,7)$. Data quality and compliance were cross-checked by the data coordinating center.

We included only initial TIs and excluded existing tracheal tube changes. We included only TIs in PICUs from institutions within the US with an existing pediatric residency program. 
We excluded TIs occurring outside the PICUs such as in emergency departments or in operating suites, since the majority of non-elective non-neonatal TIs occur in PICUs, and the database does not consistently include TIs occurring in emergency departments from each institution. We excluded ICUs where pediatric residents do not routinely rotate, such as many cardiac ICUs.

Outcome definition-The primary outcome was pediatric resident trainee participation in TI procedures as a first laryngoscopist. The secondary outcome was first attempt success in TI by pediatric resident trainees.

Pediatric airway management training structure-For each participating site, the Principal Investigator: PI (a PICU attending physician) was contacted by the investigators to identify the training structure for resident airway management. Specifically, each site PI was asked about PICU training demographics (presence of pediatric residency and PCCM fellowship programs), presence of an airway management training curriculum for pediatric residents, and details regarding the training modality (e.g. simulation-based education, hands-on TI practice in the operating suites, and didactic activities) and frequency. The PIs were also inquired about the expectation of pediatric residents to perform TIs in the PICU. Each PI was encouraged to discuss responses with other attending physicians within their division to ensure the accuracy of the response. Research Electronic Data Capture (REDCap) was used to facilitate the data collection (15). The data coordinating center contacted the site PI for their timely and full responses.

\section{Sample Size Estimation}

The sample size was calculated using a published study which showed that residents participated in nearly 30\% of intubations in 15 PICUs from July 2010 to December 2011

(2). With an assumption that TI numbers are three times larger in FY 2016 with an increased number of participating PICUs, a sample size of 4,436 (3,327 in FY 2016) was estimated to detect an absolute difference of 5\% in resident participation between FY 2011 and FY 2016 with alpha $=0.05$ and beta $=0.1$.

\section{Statistical Methods}

Our study evaluated the trend in pediatric resident participation in TIs as first laryngoscopists. The term 'trend' refers to the change over time in this manuscript. Demographics were described with proportion, and non-normally distributed continuous variables were described with median with interquartile range (IQR). Inferential statistics were performed using the Chi-squared test for categorical variables, and Wilcoxon rank-sum test for non-normally distributed variables. The trend over time was evaluated by nonparametric test for trend as previously published $(16,17)$. The impact of updated ACGME guidelines was evaluated through a multivariable logistic regression by using a generalized estimate equation (GEE) model to account for clustering within each PICU, while adjusting for patient-level characteristics and presence of a PCCM fellowship program. These patientlevel covariates were selected if they were associated with resident participation in TI in univariate analyses $(\mathrm{p}<0.1)$. Results were considered statistically significant if $\mathrm{p}$-value was less than 0.05 . 


\section{RESULTS}

\section{Tracheal intubation participation by pediatric residents over time}

A total of 9,203 TIs were reported from 25 PICUs (18 PICUs with a PCCM fellowship, 7 PICUs without a PCCM fellowship). Pediatric residents participated in 1,471 TIs (16\%).

Pediatric residents were the first laryngoscopists more often in patients who were younger and had lower Pediatric Index of Mortality (PIM2) scores, Table 1. Pediatric residents participated less often in patients with shock as an indication for intubation, history of difficult airway, or any difficult airway features.

The resident TI participation rate was significantly lower in PICUs with PCCM fellowship programs $(1,146 / 8,245,14 \%)$ vs. PICUs without fellowship programs $(325 / 958,34 \%)$, $\mathrm{p}<0.001$. The overall resident TI participation has decreased steadily over time (3.4\% each year, $\mathrm{p}<0.001$ ), as shown in Figure 1. Resident TI participation also declined significantly in PICUs with PCCM fellowship programs $(\mathrm{z}=-16.99, \mathrm{p}<0.001)$. In contrast, there was no significant change in resident TI participation in PICUs without PCCM fellowship programs $(\mathrm{z}=0.34, \mathrm{p}=0.73)$.

A multivariable analysis accounting for site-level clustering and adjusting for patient characteristics and PCCM fellowship presence demonstrated a significant yearly decline of resident TI participation [Odds ratio (OR) 0.80 for each successive year, 95\% CI: 0.70-0.91, $\mathrm{p}=0.001]$; however, the decline was not significantly affected by the change in ACGME guidelines (OR $0.86,95 \%$ CI $0.60-1.25, \mathrm{p}=0.43$ ), as shown in Table 2 . The result was similar when we limited the analysis for sites participated in the registry for the entire study period.

\section{Tracheal intubation success by pediatric residents over time}

The resident first attempt success rate was significantly higher in PICUs with PCCM fellowship $(449 / 1,146,39 \%)$ than PICUs without fellowship $(102 / 325,31 \%), p=0.01$. The resident $\mathrm{TI}$ first attempt success rate did not change over time $(\mathrm{z}=1.27, \mathrm{p}=0.21)$, regardless of the presence of PCCM fellowship, Figure 2.

\section{Airway management training curriculum for pediatric residents}

Two sites were not asked about the training curriculum, as they ended participation in NEAR4KIDS during the study period. Among 23 PICUs with a pediatric residency program, 7 PICUs (30\%) provided some form of airway management training. A systematic training curriculum for residents existed in 6/17 (35\%) PICUs with PCCM fellowship program and in 1/6 PICUs (17\%) without PCCM fellowship program ( $\mathrm{p}=0.62)$. Simulationbased training was reported as the most common educational format for resident airway management training, Table 3 . All the PICUs having training curriculum $(7 / 7,100 \%)$ included bag mask ventilation, oral or nasal airway placement, and TIs. Laryngeal mask airway placement was covered in 5/7 PICUs (71\%). Pediatric residents were expected to perform TIs in 6/23 (26\%) PICUs; this expectation was not different among PICUs with an 
airway management curriculum for residents $(2 / 7,29 \%)$ vs. PICUs without a curriculum $(4 / 16,25 \%), p=0.62$.

\section{Association between airway management training curriculum and resident participation over time}

The presence of a resident airway management training curriculum was associated with lower resident TI participation (TI in PICUs with a specific curriculum: 617/4,348: 14\% vs. TI in PICUs without a curriculum: 847/4,678: 18\%, p<0.001). The decline in resident TI participation was similar in PICUs with vs. without a resident airway management training curriculum, Figure 3. The trend for resident first attempt success was similar among PICUs with vs. without a curriculum, Figure 4.

\section{DISCUSSION}

This study examined TI participation by pediatric residents as laryngoscopists over time, especially focusing on the trend before and after the ACGME guideline change in resident non-neonatal TI requirement in 2013. Our study demonstrated that overall resident participation in TI has significantly decreased over the last 6 years. While pediatric residents participated in $16 \%$ of all TIs on average, the current resident participation rate as of fiscal year 2016 is only $8 \%$. There has been a significant yearly decline in resident TI participation, even after adjusting for patient-level characteristics including age, indication, severity score, difficult airway features and the use of neuromuscular blockade. Surprisingly, there was no significant difference in resident TI participation before and after ACGME guideline change in resident non-neonatal TI requirement. After stratifying by PCCM fellowship program presence, the decline in resident TI participation was only significant in PICUs with PCCM fellowship. Interestingly, we found no difference in the downward trend of resident TI participation among PICUs with a resident airway management curriculum versus those without it. Furthermore, the expectation for residents to perform TI and the presence of airway management training curriculum were not concordant, suggesting a mismatch in the existing training curriculum and actual resident trainee TI practice.

Our results demonstrate a significant and steady decline in resident TI participation in PICUs since 2010 to date regardless of the ACGME guideline change. The reasons for this decline are most likely multifactorial. First, it is possible that the non-neonatal TI requirement for pediatric residents might have been perceived as unrealistic in many PICUs even before the ACGME guideline change. Second, there is a domino effect, i.e., the current decrease in resident TI participation likely perpetuates further decreases in future resident TI participation. The majority of TI opportunities are needed to be given to PCCM fellows, in part due to their inability to attain non-neonatal TI skills during their pediatric residency. This could help explain the difference in resident TI participation and decline rate between PICUs with vs. without PCCM fellowships. Recent increase in the use of non-invasive ventilation (NIV) might have also reduced the total number of TIs overall (18-20). Third, the PICUs with PCCM fellowships are in larger academic centers that are usually staffed with multidisciplinary frontline providers (e.g. nurse practitioners, physician assistants, and respiratory therapists) who require ongoing training to perform TIs in PICUs. This might 
further contribute to the rapid decline in resident participation in PICUs with PCCM fellowship programs. Fourth, our ongoing multi-center TI quality improvement intervention might affect attending physicians' decision to allow residents to participate in TIs in PICUs, because the intervention emphasized the importance of provider TI skill competence to be matched to patient risk and level of technical difficulty (21). Lastly, the duty working hours for the residents might be also one of the contributing factors for more limited procedural experience (22-25).

Learning TI skills requires structured training. Previously published studies from pediatric and neonatal ICUs demonstrated that pediatric resident clinical exposure is no longer sufficient to provide training to achieve procedural competence. Anesthesiology literature with novice learners reported that in order to achieve $90 \%$ probability of TI success, one must participate in approximately $40-50$ TIs $(26,27)$. More recent studies demonstrated that there is substantial variability among individual trainees in achieving TI procedural competence, making a universal recommendation for the number of TIs difficult (28-30). Individual procedural competence assessment using cumulative sum (CUSUM) method might be more appropriate in the future $(28,30)$.

The TI first attempt success rate of pediatric residents did not change significantly over the study period. We speculate that this might be due to a patient as well as a provider selection bias, i.e., more acute care oriented residents with previous TI experience might be preferentially given more TI opportunities for low risk patients. Interestingly, the resident success rate in PICUs with PCCM fellowship was significantly higher as compared to PICUs without PCCM fellowship. It may be that residents are more restricted, and only those who have demonstrated some proficiency may be allowed to participate in TIs in these academic pediatric ICUs where routine resident participation is less expected. This process might have been even more emphasized when TI quality improvement airway bundle checklists were implemented in several PICUs (21). This might also explain the nonsignificant increase in TI success rate in PICUs without PCCM fellowship programs. It is also worth noticing that the first attempt success rate was low (i.e., less than 40\%) overall, even when pediatric residents attempted to intubate patients with lower PIM2 scores and no history of difficult airway.

Responses from site PIs revealed that $26 \%$ of PICUs expect their pediatric residents to perform TI in the PICU. One of the interesting findings was that this expectation was not concordant with the presence of an airway management training curriculum for residents. Moreover, the decline in the participation rate was similar across all PICUs, both with and without resident airway management training curricula. This can be explained by the finding that presence of an airway training curriculum was more common in academic PICUs with PCCM fellowship, where resident participation in TI is either low or not expected. This discrepancy between expectation for resident TI participation and training curriculum presence should be narrowed in the future. Specifically, there may be a need for a resident airway management training curriculum in the PICUs where they are expected to participate in TIs. Alternatively, in the PICUs where residents TIs are not expected, resident airway training curriculum might better focus on basic skills such as identification of potential 
difficult airway, bag mask ventilation skills, oral and nasal airway placement and laryngeal mask airway placement.

Simulation-based education was the dominant training modality in all of the PICUs with resident airway management training curricula, whereas didactic sessions were also provided in 5/7 PICUs. Strikingly, only 1 PICU used online-based education as a training modality. Sawyer recently published an educational pedagogical framework for procedural skills training, the Learn-See-Practice-Prove-Do-Maintain Model (31). Previous studies have demonstrated improved clinical performance in several procedures after a simulation-based training with deliberate practice and mastery learning approach $(32,33)$. During the "Prove" step in this framework, learners are required to achieve mastery in simulation before attempting the procedure on a patient. To structure TI training curricula for residents, this multi-step model with didactic and online-based education followed by simulation with mastery learning approach might be considered.

This study has several limitations. First, all TI data are self-reported by sites. To minimize any reporting biases and inaccuracy, a site-specific compliance plan has been in place and the data coordinating center crosschecks the trend and accuracy. However, we cannot completely eliminate the possibility of reporting bias. Secondly, we included only 25 PICUs in our study, which represents less than $10 \%$ of the PICUs across the US (>400 PICUs), this may limit the generalizability of our findings (34). Also, some residents might have performed TIs outside PICUs (emergency room, or operating room under supervision), which were not included in our study. Lastly, the main investigators inquired about the airway management training curriculum from the NEAR4KIDS site PIs who are PICU attending physicians engaged in airway management education and quality improvement. The reported information might not accurately describe their complete PICU practice, especially in this topic, if their teaching styles vary between them and their colleagues.

\section{CONCLUSION}

Pediatric resident participation in TIs as first laryngoscopists has declined significantly from 2010 to 2016; specifically in the PICUs with PCCM fellowship programs. The 2013 ACGME requirement change did not affect pediatric resident TI participation, after accounting for site-level clustering and adjusting for patient characteristics and PCCM fellowship presence. The first TI attempt success rate of pediatric residents did not change significantly over time. Only a quarter of PICUs have an airway management training curriculum for residents, and the presence of a training curriculum did not match the expectation of residents to perform TIs.

\section{Supplementary Material}

Refer to Web version on PubMed Central for supplementary material.

\section{Acknowledgments}

Financial Support/COI: 
This study was supported by Agency for Healthcare Research and Quality: AHRQ R03HS021583, AHRQ R18HS022464, R18HS024511 and Endowed Chair, Critical Care Medicine, The Children's Hospital of Philadelphia

Natalie Napolitano, Akira Nishisaki and Vinay Nadkarni were supported by grants AHRQ R18HS022464, R18HS024511, and AHRQ R03HS021583.

Natalie Napolitano also received grants from Aerogen, Nihon Kohden, Philips/Respironics, Draeger Medical, CVS Health; Consulting/Speaking Agreements with: Aerogen, Draeger, Actuated Medical, GeNO LLC, Smiths Medical.

We thank Hayley Buffman, MPH for administrative support. We also thank Stephanie Tuttle, MBA for her administrative leadership and support of this project throughout.

\section{Abbreviations}

ACGME Accreditation Council for Graduate Medical Education

CI Confidence Interval

FY Fiscal Year

GEE Generalized estimate equation

IQR Inter-quartile range

IRB Institutional Review Board

NEAR4KIDSVational Emergency Airway Registry for Children

OR Odds Ratio

PALISI Pediatric Acute Lung Injury and Sepsis Investigators

PCCM Pediatric Critical Care Medicine

PGY Postgraduate year

PICU Pediatric Intensive Care Unit

PIM2 Predicted Index of Mortality 2

TI Tracheal Intubation

US United States

\section{References}

1. Nishisaki A, Turner DA, Brown CA, et al. A National Emergency Airway Registry for children: landscape of tracheal intubation in 15 PICUs. Crit Care Med. 2013; 41(3):874-85. [PubMed: 23328260]

2. Sanders RC, Giuliano JS, Sullivan JE, et al. Level of trainee and tracheal intubation outcomes. Pediatrics. 2013; 131(3):e821-8. [PubMed: 23400606]

3. Nishisaki A, Donoghue AJ, Colborn S, et al. Effect of just-in-time simulation training on tracheal intubation procedure safety in the pediatric intensive care unit. Anesthesiology. 2010; 113(1):21423. [PubMed: 20526179] 
4. Ben-Isaac E, Keefer M, Thompson M, et al. Assessing the utility of procedural training for pediatrics residents in general pediatric practice. J Grad Med Educ. 2013; 5(1):88-92. [PubMed: 24404233]

5. ACGME (Accreditation Council for Graduate Medical Education). [Accessed October 11, 2016] Program Requirements for Graduate Medical Education in Pediatrics. Available at: https:// www.acgme.org/Portals/0/PFAssets/ProgramRequirements/320_pediatrics_2016.pdf.; ACGME approved: September 30, 2012; effective: July 1, 2013. Revised and effective: July 1, 2015. Revised and effective: July 1, 2016

6. Nishisaki A, Ferry S, Colborn S, et al. Characterization of tracheal intubation process of care and safety outcomes in a tertiary pediatric intensive care unit. Pediatr Crit Care Med. 2010; 12(6):1-6.

7. Rehder KJ, Giuliano JSJ, Napolitano N, et al. Increased Occurrence of Tracheal IntubationAssociated Events During Nights and Weekends in the PICU. Crit Care Med. 2015; 43(12):266874. [PubMed: 26465221]

8. Grunwell JR, Kamat PP, Miksa M, et al. National Emergency Airway Registry for Children (NEAR4KIDS) and the Pediatric Acute Lung Injury and Sepsis (PALISI) Network. Trend and Outcomes of Video Laryngoscope Use Across PICUs. Pediatr Crit Care Med. 2017; 18(8):741-49. [PubMed: 28492404]

9. Parker MM, Nuthall G, Brown C 3rd, et al. Pediatric Acute Lung Injury and Sepsis Investigators (PALISI) Network. Relationship Between Adverse Tracheal Intubation Associated Events and PICU Outcomes. Pediatr Crit Care Med. 2017; 18(4):310-318. [PubMed: 28198754]

10. Shiima Y, Berg RA, Bogner HR, et al. National Emergency Airway Registry for Children Investigators. Cardiac Arrests Associated With Tracheal Intubations in PICUs. Crit Care Med. 2016; 44(9):1675-82. [PubMed: 27071070]

11. Sanders RC, Nett ST, Davis KF, et al. National Emergency Airway Registry for Children NEAR4KIDS Investigators; Pediatric Acute Lung Injury and Sepsis Investigators Network. Family Presence During Pediatric Tracheal Intubations. JAMA Pediatr. 2016; 170(3):e154627. [PubMed: 26954533]

12. Tarquinio KM, Howell JD, Montgomery V, et al. National Emergency Airway Registry for Children; Pediatric Acute Lung Injury and Sepsis Investigators Network. Current Medication Practice and Tracheal Intubation Safety Outcomes From a Prospective Multicenter Observational Cohort Study. Pediatr Crit Care Med. 2015; 16(3):210-18. [PubMed: 25581629]

13. Graciano AL, Tamburro R, Thompson AE, et al. Incidence and associated factors of difficult tracheal intubations in pediatric ICUs: a report from National Emergency Airway Registry for Children: NEAR4KIDS. Intensive Care Med. 2014; 40(11):1659-69. [PubMed: 25160031]

14. Nett S, Emeriaud G, Jarvis JD, et al. Site-level variance for adverse tracheal intubation-associated events across 15 North American PICUs: a report from the national emergency airway registry for children. Pediatr Crit Care Med. 2014; 15(4):306-13. [PubMed: 24691538]

15. Harris PA, Taylor R, Thielke R, Payne J, et al. Research electronic data capture (REDCap)-A metadata-driven methodology and workflow process for providing translational research informatics support. J Biomed Inform. 2009; 42(2):377-81. [PubMed: 18929686]

16. Sasieni PD. Stratified test for trend across ordered groups. Stata Technical Bulletin. 1996; 33:2427. [Access verified on September 08, 2017] www.stata.com/products/stb/journals/stb33.pdf.

17. Sasieni PD, Stepniewska KA, Altman DG. Test for trend across ordered groups revisited. Stata Technical Bulletin. 1996; 32:27-29. [Access verified on September 08, 2017] www.stata.com/ products/stb/journals/stb32.pdf.

18. Hull J. The value of non-invasive ventilation. Arch Dis Child. 2014; 99(11):1050-54. [PubMed: 24966355]

19. James CS, Hallewell CP, James DP, et al. Predicting the success of non-invasive ventilation in preventing intubation and re-intubation in the paediatric intensive care unit. Intensive Care Med. 2011; 37(12):1994-2001. [PubMed: 21983628]

20. Morris JV, Ramnarayan P, Parslow RC, et al. Outcomes for Children Receiving Noninvasive Ventilation as the First-Line Mode of Mechanical Ventilation at Intensive Care Admission: A Propensity Score-Matched Cohort Study. Crit Care Med. 2017; 45(6):1045-1053. [PubMed: 28328654] 
21. Li S, Rehder KJ, Giuliano JS, et al. National Emergency Airway Registry for Children (NEAR4KIDS) Investigators; Pediatric Acute Lung Injury and Sepsis Investigator PALISI Network Investigators. Development of a Quality Improvement Bundle to Reduce Tracheal Intubation-Associated Events in Pediatric ICUs. Am J Med Qual. 2016; 31(1):47-55. [PubMed: 25143411]

22. Ahmed N, Devitt KS, Keshet I, et al. A systematic review of the effects of resident duty hour restrictions in surgery: impact on resident wellness, training, and patient outcomes. Ann Surg. 2014; 259(6):1041-1053. [PubMed: 24662409]

23. Jamal MH, Rousseau MC, Hanna WC, et al. Effect of the ACGME duty hours restrictions on surgical residents and faculty: a systematic review. Acad Med. 2011; 86(1):34-42. [PubMed: 21099662]

24. Jamal MH, Wong S, Whalen TV. Effects of the reduction of surgical residents' work hours and implications for surgical residency programs: a narrative review. BMC Med Educ. 2014; 14(1):S14. [PubMed: 25560685]

25. Connors RC, Doty JR, Bull DA, et al. Effect of work-hour restriction on operative experience in cardiothoracic surgical residency training. J Thorac Cardiovasc Surg. 2009; 137(3):710-13. [PubMed: 19258094]

26. Konrad C, Schüpfer G, Wietlisbach M, et al. Learning manual skills in anesthesiology: Is there a recommended number of cases for anesthetic procedures? Anesth Analg. 1998; 86(3):635-639. [PubMed: 9495429]

27. Mulcaster JT, Mills J, Hung OR, et al. Laryngoscopic intubation: learning and performance. Anesthesiology. 2003; 98(1):23-27. [PubMed: 12502974]

28. Ishizuka M, Rangarajan V, Sawyer TL, et al. The Development of Tracheal Intubation Proficiency Outside the Operating Suite During Pediatric Critical Care Medicine Fellowship Training: A Retrospective Cohort Study Using Cumulative Sum Analysis. Pediatr Crit Care Med. 2016; 17(7):e309-16. [PubMed: 27214591]

29. Filho DO, Rodrigues G. The Construction of Learning Curves for Basic Skills in Anesthetic Procedures: An Application for the Cumulative Sum Method. Anesth Analg. 2002; 95(2):411-16. [PubMed: 12145063]

30. Komatsu R, Kasuya Y, Yogo H, et al. Learning curves for bag-and-mask ventilation and orotracheal intubation: an application of the cumulative sum method. Anesthesiology. 2010; 112(6):1525-31. [PubMed: 20463580]

31. Sawyer T, White M, Zaveri P, et al. Learn, See, Practice, Prove, Do, Maintain. Acad Med. 2015; 90(8):1025-33. [PubMed: 25881645]

32. Ericsson KA. Deliberate practice and the acquisition and maintenance of expert performance in medicine and related domains. Acad Med. 2004; 79(10 Suppl):S70-S81.

33. McGaghie WC, Issenberg SB, Cohen ER, et al. Medical Education Featuring Mastery Learning With Deliberate Practice Can Lead to Better Health for Individuals and Populations. Acad Med. 2011; 86(11):e8-9. [PubMed: 22030671]

34. Odetola FO, Clark SJ, Freed GL, et al. A National Survey of Pediatric Critical Care Resources in the United States. Pediatrics. 2005; 115(4):e382-386. [PubMed: 15805338] 


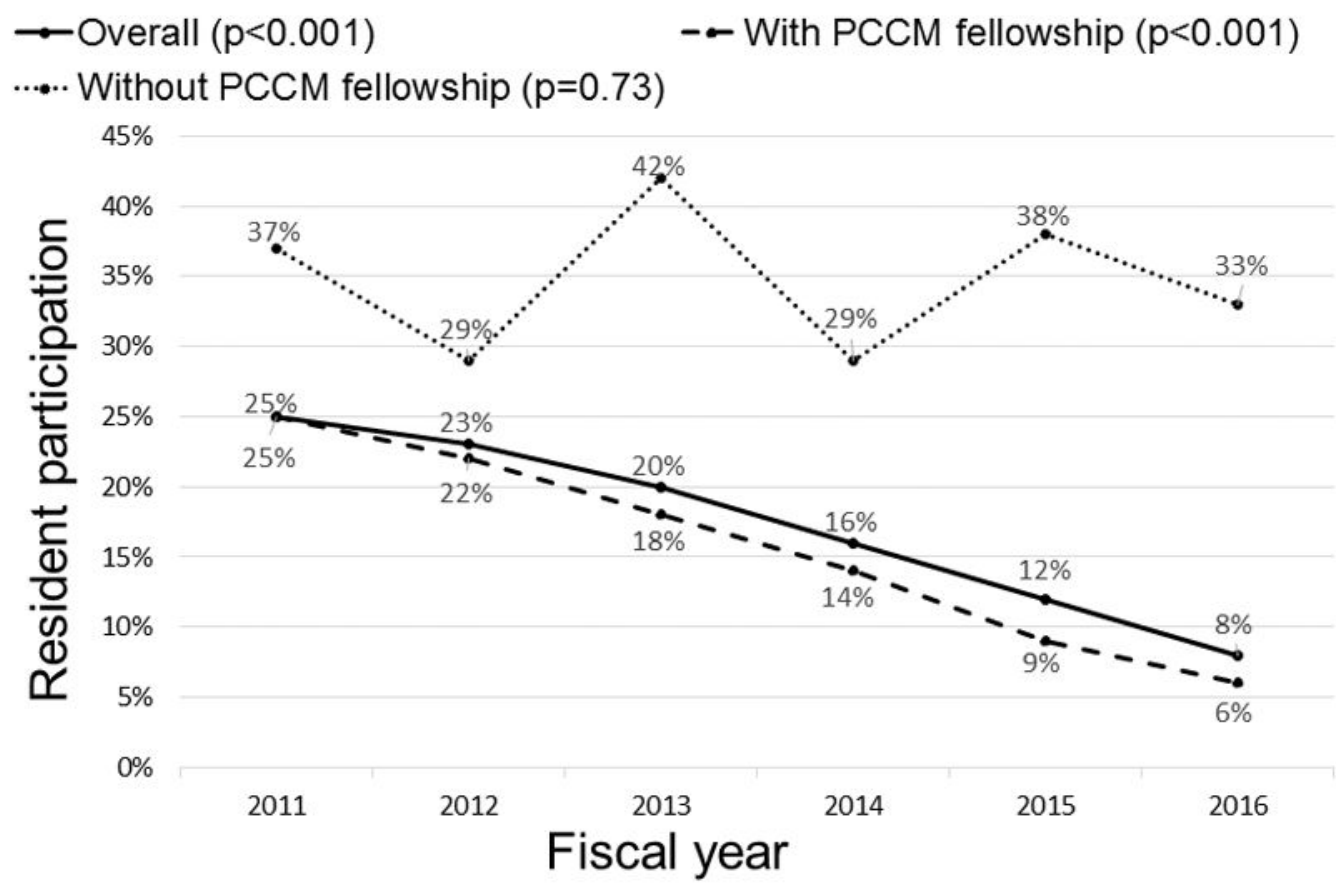

Figure 1.

Resident participation over the years

P-value was calculated by non-parametric test for trend. See Statistical Method section in the Manuscript.

PCCM denotes Pediatric Critical Care Medicine. 


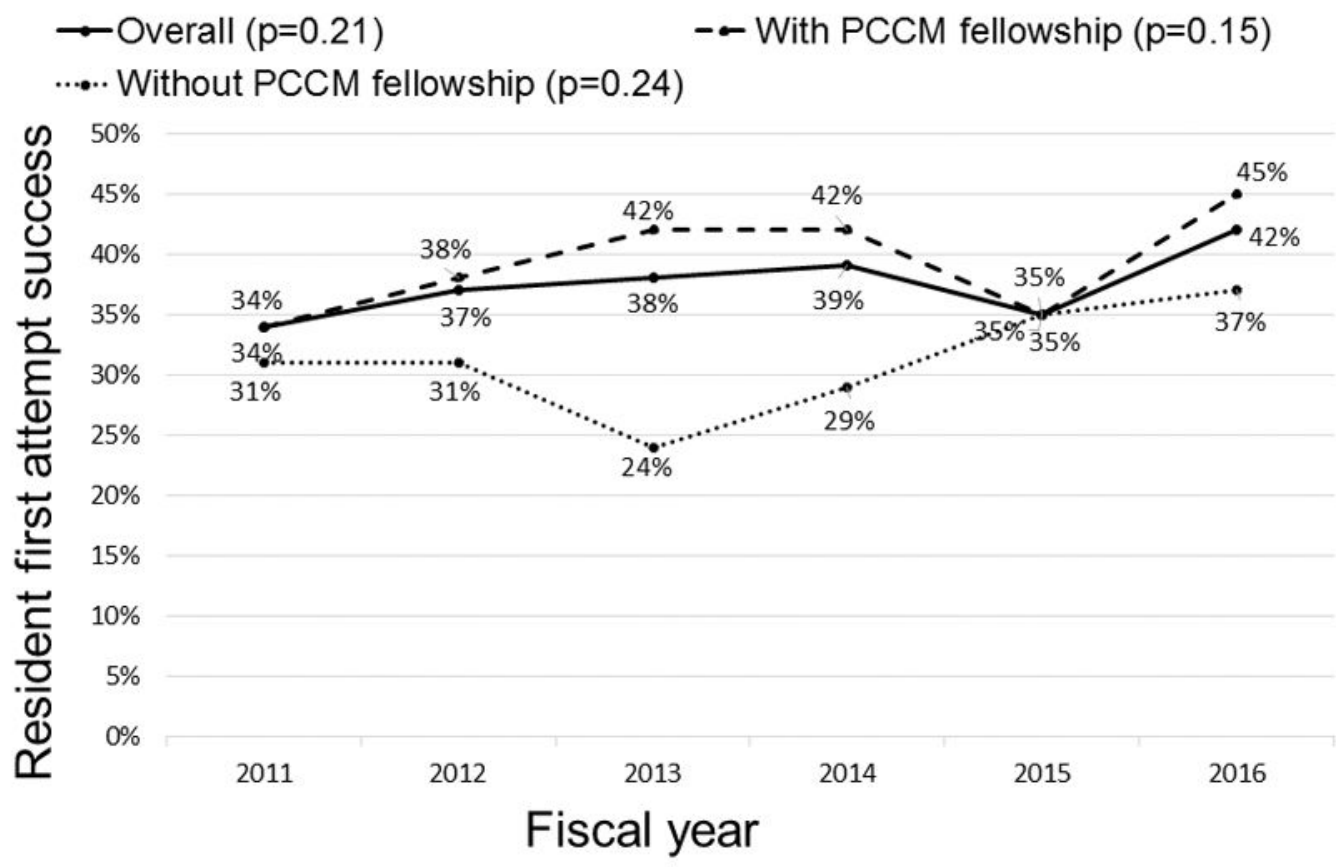

Figure 2.

Resident first attempt success rate over the years

P-value was calculated by non-parametric test for trend. See Statistical Method section in the Manuscript.

PCCM denotes Pediatric Critical Care Medicine 


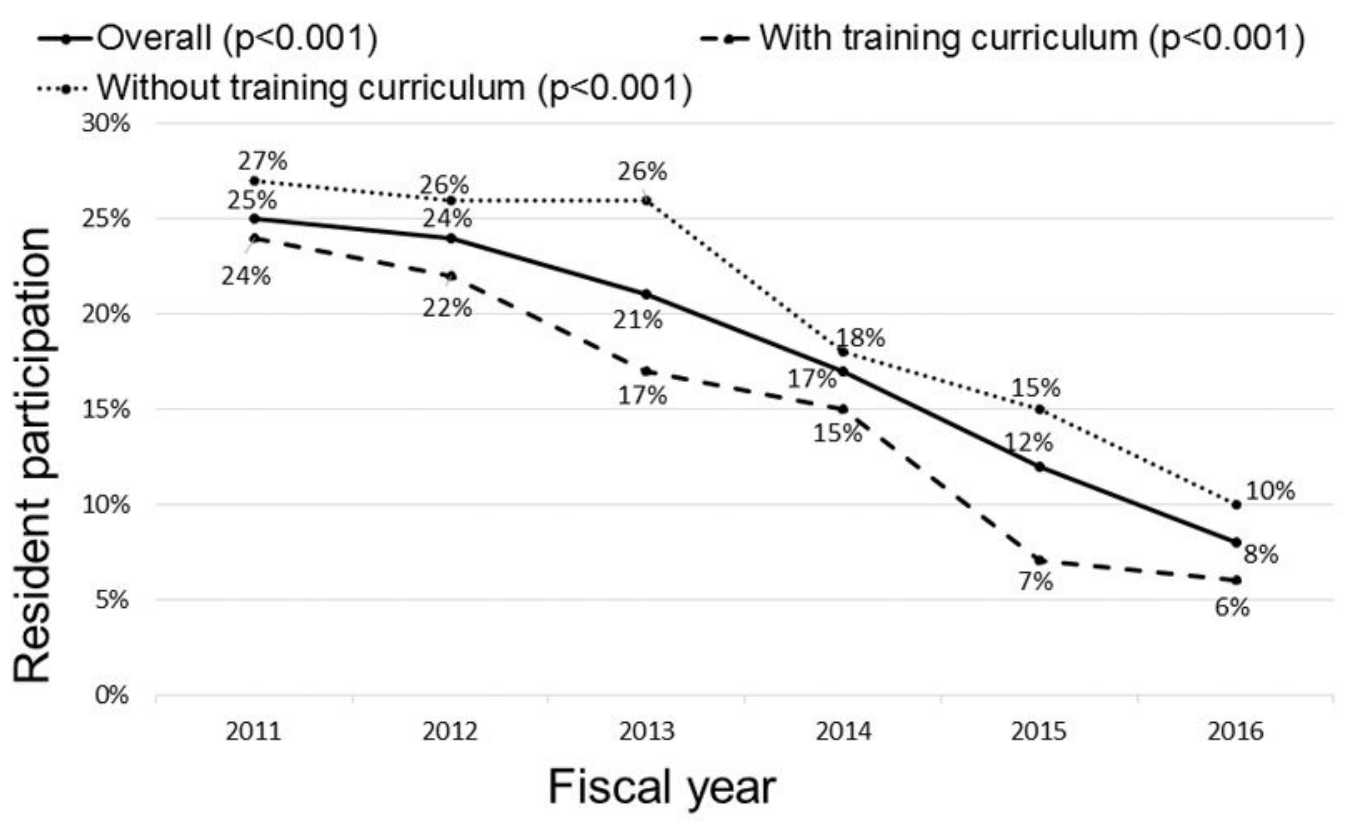

Figure 3.

Resident participation over the years stratified by airway training curriculum P-value was calculated by non-parametric test for trend. See Statistical Method section in the Manuscript. 


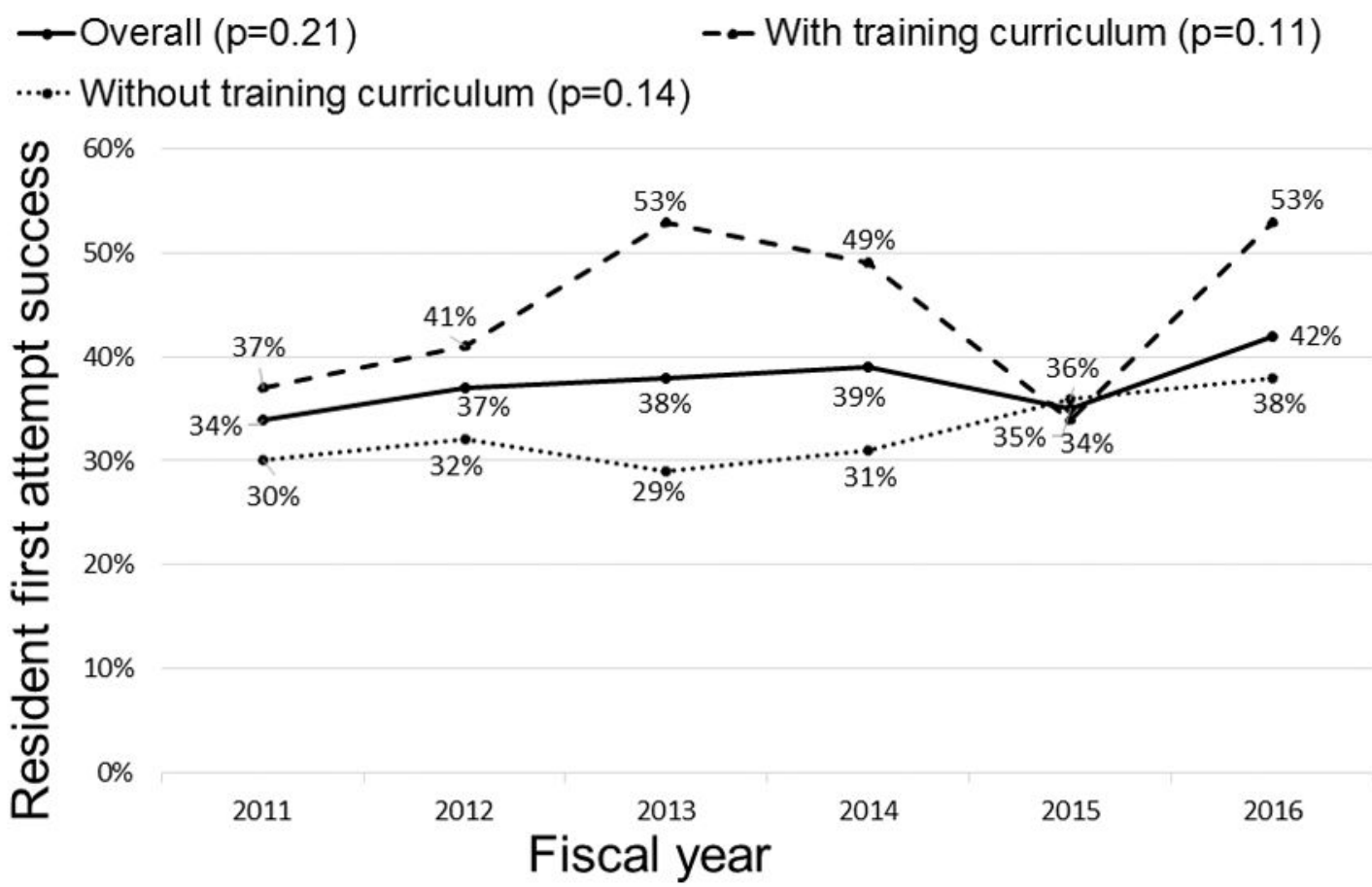

Figure 4.

Resident first attempt success rate over the years stratified by airway training curriculum P-value was calculated by non-parametric test for trend. See Statistical Method section in the Manuscript. 


\section{Table 1}

Patient demographics and practice characteristics among tracheal intubations by pediatric residents and those by other providers

\begin{tabular}{|c|c|c|c|}
\hline Variables & $\begin{array}{c}\text { Pediatric Resident as a first airway } \\
\text { provider }(n=1,471)\end{array}$ & $\begin{array}{l}\text { Other Provider as a first airway } \\
\text { provider }(n=7,732)\end{array}$ & p-value \\
\hline Age (years, median, IQR) & $1(0-7)$ & $2(0-9)$ & $<0.0001$ \\
\hline Weight (kg, median, IQR) & $10(5-23)$ & $12(6-28)$ & $<0.0001$ \\
\hline Gender (Male, n, \%) & $831(57)$ & $4,309(56)$ & 0.54 \\
\hline Diagnosis category $^{a}(\mathrm{n}, \%)$ & & & $<0.001$ \\
\hline Respiratory & $701(49)$ & $3,652(49)$ & \\
\hline Cardiac & $80(6)$ & $731(9)$ & \\
\hline Shock & $90(6)$ & $616(8)$ & \\
\hline Neurological & $364(25)$ & $1,496(20)$ & \\
\hline Trauma & $38(3)$ & $176(2)$ & \\
\hline Other & $164(11)$ & $939(12)$ & \\
\hline \multicolumn{4}{|l|}{ Indication $^{b}(\mathrm{n}, \%)$} \\
\hline Respiratory & $935(64)$ & $5,046(65)$ & 0.21 \\
\hline Procedural & $284(19)$ & $1,382(18)$ & 0.19 \\
\hline Shock & $97(7)$ & $943(12)$ & $<0.001$ \\
\hline Neurological & $204(14)$ & $995(13)$ & 0.29 \\
\hline $\operatorname{PIM} \operatorname{score}^{c}(\%$ mortality, IQR) & $1.3(0.8-4.6)$ & $2.8(0.9-6.1)$ & $<0.0001$ \\
\hline History of difficult airway (n, \%) & $141(10)$ & $1,261(16)$ & $<0.001$ \\
\hline Any difficult airway features (n, \%) & $461(31)$ & $2,670(35)$ & 0.018 \\
\hline Provider PGY level ${ }^{d}$ & & Not applicable & Not applicable \\
\hline PGY1 & $27(2)$ & & \\
\hline PGY2 & $600(43)$ & & \\
\hline PGY3 & $746(54)$ & & \\
\hline PGY4 or higher & $19(1)$ & & \\
\hline Neuromuscular blockade use (n, \%) & $1,332(91)$ & $6,741(87)$ & $<0.001$ \\
\hline \multicolumn{4}{|c|}{ Diagnosis category is missing in 156 encounters. } \\
\hline \multicolumn{4}{|c|}{$b_{\text {There may be one than one indication for each tracheal intubation. }}$} \\
\hline \multicolumn{4}{|c|}{${ }^{c}$ PIM2 denotes Predicted Index of Mortality 2 score (\%). PIM2 was available in 7,334 TI encounters. } \\
\hline \multicolumn{4}{|l|}{${ }^{d}$ PGY level was missing in 79 encounters. } \\
\hline \multicolumn{4}{|l|}{ IQR denotes interquartile range. } \\
\hline denotes post- & & & \\
\hline
\end{tabular}




\section{Table 2}

Multivariable analysis for pediatric resident as a first laryngoscopist, adjusted for patient characteristics and Pediatric Critical Care Medicine fellowship

\begin{tabular}{|lccc|}
\hline Variable & Odds Ratio (OR) & 95\% Confidence Interval & p-value \\
\hline Fiscal Year & 0.80 & $0.70-0.91$ & 0.001 \\
PCCM fellowship presence & 0.32 & $0.16-0.64$ & 0.001 \\
New ACGME guidelines ${ }^{a}$ & 0.86 & $0.60-1.24$ & 0.432 \\
Patient characteristics & & & \\
Age category & & & \\
$\quad$ Infant (less than 1 year) & Reference & & \\
$\quad$ Young child (1-7year) & 0.69 & $0.55-0.87$ & 0.001 \\
$\quad$ Child (8-17year) & 0.66 & $0.54-0.81$ & $<0.001$ \\
$\quad$ Adult ( 218 year) & 0.72 & $0.56-0.94$ & 0.016 \\
Indication & & & \\
Shock & 0.55 & $0.41-0.74$ & $<0.001$ \\
Procedure & 1.02 & $0.68-1.53$ & 0.937 \\
PIM2 score $b$ & 0.98 & $0.98-0.99$ & 0.001 \\
History of difficult airway & 0.56 & $0.45-0.70$ & $<0.001$ \\
Any difficult airway feature & 0.89 & $0.76-1.04$ & 0.151 \\
Neuromuscular blockade use & 1.38 & $1.00-1.91$ & 0.048 \\
\hline
\end{tabular}

The model was adjusted for site-level clustering by generalized estimate equation. See Method section for details.

Fiscal Year: Fiscal year 2011 (July 2010-June 2011) was used as a reference to calculate odds ratio.

${ }^{a}$ Accreditation Council for Graduate Medical Education

$b_{\text {Pediatric Index of Mortality } 2 \text { score }}$ 


\section{Table 3}

Airway management training curriculum for pediatric residents stratified by presence of pediatric critical care medicine fellowship

\begin{tabular}{|c|c|c|c|}
\hline $\operatorname{ICU}^{a}$ type & $\mathrm{ICU}^{a}$ with $\mathrm{PCCM}^{b}$ Fellowship (n=17) & $\operatorname{ICU}^{a}$ without $\underset{(\mathrm{PCCM})}{\mathrm{PCM}^{b}}$ Fellowship & Total $(n=23)$ \\
\hline Any Airway Training for residents & $6(35 \%)$ & $1(17 \%)$ & $7(30 \%)$ \\
\hline Simulation training & $6(35 \%)$ & $1(17 \%)$ & $7(30 \%)$ \\
\hline Training in the Operating Suites & $1(6 \%)$ & $0(0 \%)$ & $1(4 \%)$ \\
\hline Training using on-line materials & $1(6 \%)$ & $0(0 \%)$ & $1(4 \%)$ \\
\hline Didactic session & $4(24 \%)$ & $1(17 \%)$ & $5(22 \%)$ \\
\hline Training with Anesthesiologists & $2(12 \%)$ & $0(0 \%)$ & $2(9 \%)$ \\
\hline Training in the Emergency Department & $3(18 \%)$ & $0(0 \%)$ & $3(13 \%)$ \\
\hline
\end{tabular}

ICU- Intensive Care Unit.

$b_{\text {PCCM- pediatric critical care medicine. }}$ 